\title{
Stability of Sb-Te layered structures: First-principles study
}

\author{
K. Govaerts, ${ }^{1, *}$ M. H. F. Sluiter, ${ }^{2, \dagger}$ B. Partoens, ${ }^{3, \ddagger}$ and D. Lamoen ${ }^{1, \S}$ \\ ${ }^{1}$ EMAT, Universiteit Antwerpen, Groenenborgerlaan 171, 2020 Antwerpen, Belgium \\ ${ }^{2}$ Department of Materials Science and Engineering, $3 \mathrm{mE}$, Delft University of Technology, Mekelweg 2, 2628 CD, Delft, The Netherlands \\ ${ }^{3}$ CMT Group, Department of Physics, Universiteit Antwerpen, Groenenborgerlaan 171, 2020 Antwerpen, Belgium
}

(Received 30 August 2011; revised manuscript received 23 December 2011; published 23 April 2012)

\begin{abstract}
Using an effective one-dimensional cluster expansion in combination with first-principles electronic structure calculations we have studied the energetics and electronic properties of Sb-Te layered systems. For a $\mathrm{Te}$ concentration between 0 and 60 at. $\%$ an almost continuous series of metastable structures is obtained consisting of consecutive $\mathrm{Sb}$ bilayers next to consecutive $\mathrm{Sb}_{2} \mathrm{Te}_{3}$ units, with the general formula $\left(\mathrm{Sb}_{2}\right)_{n}\left(\mathrm{Sb}_{2} \mathrm{Te}_{3}\right)_{m}$ $(n, m=1,2, \ldots)$. Between 60 and 100 at. \% no stable structures are found. We account explicitly for the weak van der Waals bonding between $\mathrm{Sb}$ bilayers and $\mathrm{Sb}_{2} \mathrm{Te}_{3}$ units by using a recently developed functional, which strongly improves the interlayer bonding distances. At $T=0 \mathrm{~K}$, no evidence is found for the existence of two separate single-phase regions $\delta$ and $\gamma$ and a two-phase region $\delta+\gamma$. Metastable compounds with a Te concentration between 0 and 40 at. $\%$ are semimetallic, whereas compounds with a Te concentration between 50 and 60 at. \% are semiconducting. Compounds with an odd number of $\mathrm{Sb}$ layers are metallic and have a much higher formation energy than those with an even number of consecutive Sb layers, thereby favoring the formation of Sb bilayers.
\end{abstract}

DOI: 10.1103/PhysRevB.85.144114

PACS number(s): 61.50.-f, 71.20.-b, 31.15.A-

\section{INTRODUCTION}

$\mathrm{Sb}$-Te alloys exhibit several interesting properties. $\mathrm{Sb}_{2} \mathrm{Te}_{3}$, which is the most stable compound, is not only known as a topological insulator ${ }^{1,2}$ but also as a thermoelectric ${ }^{3}$ and as one of the Ge-Sb-Te based phase change (PC) materials used for optical data storage (CD, DVD, Blu-ray disk). ${ }^{4}$ In fact compounds with a composition close to the "eutectic" composition $\mathrm{Sb}_{2} \mathrm{Te}$ are considered as potential candidates for replacing the existing Flash technology in nonvolatile random access memory (RAM). ${ }^{5}$ PC materials reversibly switch between a crystalline (a binary " 1 ") and an amorphous (a binary "0") state under the application of an electrical pulse. The difference in electrical resistivity is then used to identify both phases. However, the current that is needed to heat the PC material as well as the melting and crystallization temperature all depend strongly on the Sb content and on the doping conditions. Therefore it is important to have a detailed knowledge of the $\mathrm{Sb}$-Te structure as a function of the $\mathrm{Sb}$ content. Moreover, since the crystalline-amorphous transition in RAM cells is triggered by an electrical current, it is also important to know the relation between structural and electronic properties.

$\mathrm{Sb}_{2} \mathrm{Te}_{3}$ has a rhombohedral layered structure with space group $R \overline{3} \mathrm{~m}$ in which alternating layers of Te and $\mathrm{Sb}$ are stacked with a sequence Te-Sb-Te-Sb-Te along the [0001] direction of the conventional hexagonal unit cell as shown on the left in Fig. 1. The layers follow a cubic-close-packed ABCABC... sequence along this [0001] direction. The hexagonal unit cell contains 15 layers with lattice parameters $a=4.264 \AA$ and $c=30.458 \AA^{6}{ }^{6}$ The phase diagram of Sb-Te (Ref. 7) shows that besides the stable $\mathrm{Sb}_{2} \mathrm{Te}_{3}$ phase (at 60 at. \% Te), two single-phase regions with broad stoichiometry range from 18 to 37 and 42 to 45 at. \% Te exist, which are denoted as $\delta$ and $\gamma$, respectively. Often these phases are also referred to as $\mathrm{Sb}_{2} \mathrm{Te}$ and $\mathrm{SbTe}$. In between the $\delta$ and $\gamma$ phases there is a two-phase region $\delta+\gamma$. The Te-rich side (more than 60 at. $\% \mathrm{Te}$ ) of the phase diagram shows a clear phase separation of bulk Te and $\mathrm{Sb}_{2} \mathrm{Te}_{3}$.
In the past decades ${ }^{8-14}$ several Sb-rich compounds (mainly in the $\delta$ phase) have been studied and characterized and it turns out that all known $\mathrm{Sb}$-Te compounds with an $\mathrm{Sb}$ concentration above 40 at. $\%$ are composed of bilayers of $\mathrm{Sb}$ and $\mathrm{Sb}_{2} \mathrm{Te}_{3}$ units and can be represented by the general formula $\left(\mathrm{Sb}_{2}\right)_{n}\left(\mathrm{Sb}_{2} \mathrm{Te}_{3}\right)_{m}$. The investigated compounds had a very similar in-plane lattice parameter $a$, but the $c$ parameter varied strongly with the amount of $\mathrm{Sb}$ and $c$ values up to $100 \AA$ have been found. ${ }^{11}$ In particular, the $\mathrm{Sb}_{2} \mathrm{Te}$ structure of the $\delta$ phase has a hexagonal unit cell containing 9 layers: $\mathrm{Sb}_{4}\left(\mathrm{Sb}_{2} \mathrm{Te}_{3}\right)$, with $a=4.272 \AA$ and $c=17.633 \AA,{ }^{15}$ shown in the middle in Fig. 1, and the SbTe structure of the $\gamma$ phase has a hexagonal unit cell containing 12 layers: $\mathrm{Sb}_{2}\left(\mathrm{Sb}_{2} \mathrm{Te}_{3}\right)_{2}$, with $a=4.26 \AA$ and $c=23.9 \AA,^{8}$ shown on the right in Fig. 1 . The bonding between adjacent $\mathrm{Sb}$ bilayers, between adjacent $\mathrm{Sb}_{2} \mathrm{Te}_{3}$ units, and between $\mathrm{Sb}$ bilayers and $\mathrm{Sb}_{2} \mathrm{Te}_{3}$ units is of the van der Waals type.

In this paper we study systematically the relation between composition and structure from first principles. However in order to establish a one-to-one correspondence between the composition and the structure of the $\mathrm{Sb}_{1-x} \mathrm{Te}_{x}$ alloy for any value of $x$ (here and in the following $x$ will denote the Te concentration), one needs a systematic methodology which allows for a fast and accurate calculation of total energies and for an easy discrimination between stable and unstable phases. In this work we use the cluster expansion (CE) method me,17 $^{16}$ in combination with first-principles electronic structure calculations to study the energetics, and structural and electronic properties of arbitrary stackings of Sb and Te layers. Moreover the combination of both methods yields insight into the microscopic origin of the stability of the layered Sb-Te phases.

The rest of the paper is organized as follows. The details of the CE implementation are given in Sec. II and in Sec. III we give some technical details on the first-principles calculations. The results of the CE and details of the optimized structures are discussed in Sec. IV A. The electronic properties of the obtained (meta)stable states in the $\mathrm{Sb}$-Te system are addressed in Sec. IV B. Finally we summarize our results in Sec. V. 


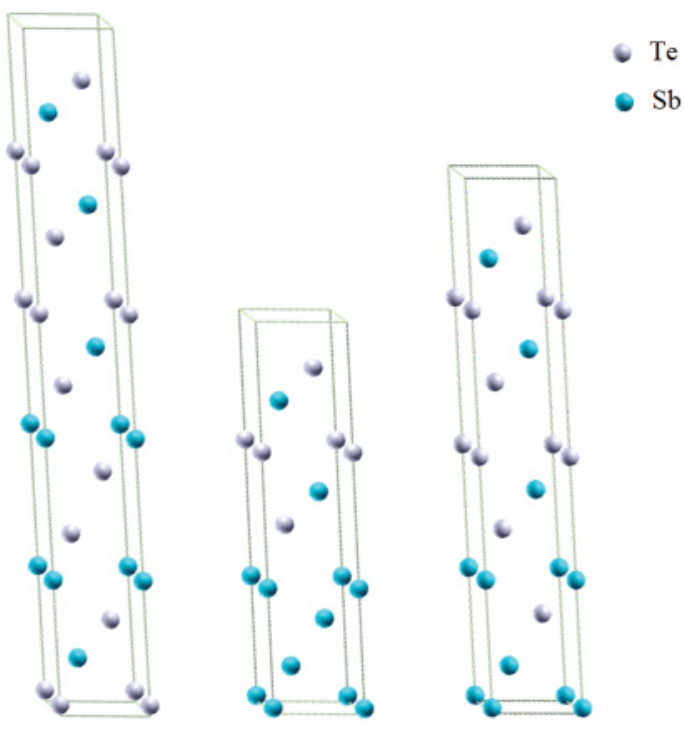

FIG. 1. (Color online) Experimentally observed structures in SbTe alloys. The hexagonal unit cell of $\mathrm{Sb}_{2} \mathrm{Te}_{3}$ (left) contains three units of Te-Sb-Te-Sb-Te. The cell contains 15 layers, with the number of layers always a multiple of 3 to fulfill the $\mathrm{ABCABC}$ sequence. The $\delta$ and $\gamma$ phases are often referred to as $\mathrm{Sb}_{2} \mathrm{Te}$ (middle) and $\mathrm{SbTe}$ (right), respectively.

\section{CLUSTER EXPANSION METHOD}

For a given lattice with sites being occupied by $\mathrm{Sb}$ or Te atoms, configurational disorder can easily be accounted for by the introduction of an occupation variable $\sigma_{i}$ which takes the value 1 or 0 when the lattice site $i$ is occupied by $\mathrm{Te}$ or $\mathrm{Sb}$, respectively. Since we only consider the ordering of $\mathrm{Sb}$ and Te layers, we have an effective one-dimensional system and the site index $i$ labels the different layers. The configuration of a structure can then be specified by a vector $\bar{\sigma}=\left\{\sigma_{1}, \sigma_{2}, \ldots, \sigma_{N}\right\}$, when the unit cell contains $N$ layers. The formation energy of the $\mathrm{Sb}_{1-x} \mathrm{Te}_{x}$ alloy is defined by

$$
E_{\mathrm{form}}(\bar{\sigma})=E(\bar{\sigma})-[x E(\mathrm{Te})+(1-x) E(\mathrm{Sb})],
$$

where $E(\bar{\sigma})$ is the total energy of configuration $\bar{\sigma}$ per atom, and $E(\mathrm{Te})$ and $E(\mathrm{Sb})$ are the energies of pure Te and pure $\mathrm{Sb}$. The CE is based on a generalization of the well-known Ising model and parametrizes the formation energy as a function of the occupation variables $\sigma^{16}$ :

$$
E_{\text {form }}(\bar{\sigma})=\sum_{\alpha} V_{\alpha} \xi_{\alpha}(\bar{\sigma})
$$

where $\alpha$ is a cluster of lattice sites, for example an empty cluster, a cluster with one lattice point, a cluster with nearestneighbor lattice points, a triangle,..., and

$$
\xi_{\alpha}(\bar{\sigma})=\left\langle\prod_{i \in \alpha} \sigma_{i}(\bar{\sigma})\right\rangle
$$

are the correlation functions, with the average taken over all clusters of the same type. The expansion coefficients $V_{\alpha}$ are the so-called effective cluster interactions (ECIs). The ECIs give the relative importance of each cluster $\alpha$. The correlation functions $\xi_{\alpha}$ play the roll of basis functions; they form a complete and orthonormal basis. The CE of Eq. (2) is in principle exact when all clusters are considered, but in practice often only a limited number of clusters is necessary to adequately represent the energy. The ECIs are found by solving Eq. (2) for the ECIs using the energies calculated from first principles for a relatively small number of configurations by using a least-squares procedure (a generalization of the Connolly-Williams approach ${ }^{18}$ ). Once the ECIs are known, Eq. (2) can be used to calculate the formation energy of an arbitrary configuration (structure) at any concentration.

In the case of the layered compounds of Sb-Te, a structure is completely defined by a certain sequence of layers, consisting of only $\mathrm{Sb}$ atoms or only $\mathrm{Te}$ atoms. It is sufficient to consider only one-dimensional clusters, because the correlation functions $\xi_{\alpha}$ do not depend on the specific position of an atom within the layer, but only on the plane in which it is residing. The energies of a very large number of structures is then computed with the $\mathrm{CE}$ and the convex hull formed by the stable structures is determined over the whole concentration range. The convex hull, which is the line connecting the stable states, determines whether a compound $\mathrm{Sb}_{1-x} \mathrm{Te}_{x}$ is thermodynamically stable or whether a two-phase region will emerge.

In Ref. 19 it was shown that all subclusters of a considered cluster must be included in order to make the $\mathrm{CE}$ invariant with regard to the definition of the occupation variable. To determine which clusters (with all their subclusters) should be used in the $\mathrm{CE}$, we use the leave-one-out-cross validation (LOOCV), ${ }^{20,21}$ which is defined by

$$
\mathrm{LOOCV}=\sqrt{\frac{1}{S} \sum_{j=1}^{S}\left[E_{\mathrm{form}}\left(\bar{\sigma}_{j}\right)-E_{\mathrm{form}}^{*}\left(\bar{\sigma}_{j}\right)\right]^{2}}
$$

with $S$ the number of structures, $E_{\text {form }}\left(\bar{\sigma}_{j}\right)$ the $a b$ initio computed formation energy of configuration $\bar{\sigma}_{j} \cdot E_{\text {form }}^{*}\left(\bar{\sigma}_{j}\right)$ is obtained from a $\mathrm{CE}$ in which the ECIs are calculated from a least-squares fit based on all structures with the exclusion of the $j$ th structure. Optimization of the LOOCV over all permutations of cluster combinations yields a "best" $\mathrm{CE}$. If this CE predicts new groundstates-structures with formation energy on or below the convex hull-then these energies should be calculated from first-principles and added to the dataset of energies. With this new dataset a new CE can be found and so on, until no new ground states are found.

To speed up the convergence, a larger weight can be attributed to the structures on or near the convex hull. In this work, we have chosen the following weight factor ${ }^{22}$ :

$$
w\left(\bar{\sigma}_{j}\right)=\frac{1}{1+\omega\left(\frac{d\left(\bar{\sigma}_{j}\right)}{\langle d\rangle}\right)},
$$

where $d\left(\bar{\sigma}_{j}\right)$ is the energy difference of the formation energy of structure $\bar{\sigma}_{j}$ and the line of the convex hull, $\langle d\rangle$ is the mean value of these energy differences taken from all structures. Here we have chosen $\omega=1$.

As we discuss in more detail below, we also considered a ternary $\mathrm{CE}$, in which the occupation variable for Te remains 1 , but the occupation variable for $\mathrm{Sb}$ switches from 0 to -1 if the $\mathrm{Sb}$ layer is a part of a sequence of an even number of $\mathrm{Sb}$ 
layers. The $\mathrm{CE}$ then becomes

$$
E_{\text {form }}(\bar{\sigma})=\sum_{\alpha} \sum_{\tau} V_{\alpha}^{\tau} \xi_{\alpha}^{\tau}(\bar{\sigma})
$$

with correlation coefficients

$$
\xi_{\alpha}^{\tau}(\bar{\sigma})=\left\langle\prod_{i \in \alpha} \varphi_{\tau_{i}}\left(\sigma_{i}(\bar{\sigma})\right)\right\rangle
$$

and where the index $\tau$ runs over the basis functions, which are given by

$\varphi_{0}\left(\sigma_{i}\right)=1, \quad \varphi_{1}\left(\sigma_{i}\right)=\sqrt{\frac{3}{2}} \sigma_{i}, \quad \varphi_{2}\left(\sigma_{i}\right)=-\sqrt{2}\left(1-\frac{3}{2} \sigma_{i}\right)^{2}$.

This set forms a complete and orthonormal basis. ${ }^{23}$

\section{COMPUTATIONAL DETAILS}

The first-principles calculations have been performed within the density-functional theory (DFT) formalism as implemented in the VASP code. ${ }^{24,25}$ We used the all-electron projector augmented wave (PAW) with the generalized gradient approximation of Perdew-Burke-Ernzerhof (PBE). ${ }^{26}$ In order to account for the effect of London dispersion forces we use the van der Waals density functional (vdW-DF) as implemented in the VASP code. ${ }^{27}$ In particular we have used the so-called optB86b-vdW functional, which provides superior values for the lattice constants in comparison with other vdW-DF functionals (for a detailed discussion on this matter we refer to Ref. 27). For total-energy calculations and the optimization of the structures we used a plane-wave cutoff value of $250 \mathrm{eV}$. For the Brillouin-zone integration we used a $16 \times 16 \times \ell$ grid, ${ }^{28}$ with $\ell$ depending on the $c$ lattice parameter of the hexagonal unit cell, for three layers $\ell=8$, for six layers $\ell=4$, for nine layers $\ell=3, \ldots$ Increasing the cutoff to $350 \mathrm{eV}$ and the number of $k$ points shows that our results are converged within $0.5 \mathrm{meV} /$ atom. Both lattice parameters and atom coordinates are relaxed. For the electronic structure calculation we considered the results as converged when the energy difference between two successive steps was smaller than $10^{-5} \mathrm{eV}$ and for the geometry optimization we considered a convergence criterium of $10^{-4} \mathrm{eV}$ between two successive steps. The forces on the atoms of the relaxed structures were less than $10^{-2} \mathrm{eV} / \AA$.

\section{RESULTS AND DISCUSSION}

\section{A. Structural stability}

In this section we discuss the results obtained from the one-dimensional $\mathrm{CE}$. The underlying lattice is the fcc lattice with its ABCABC... stacking of the [111] planes, each plane containing only $\mathrm{Sb}$ or Te atoms.

As a first step we kept the atomic positions fixed to equidistant layers and performed a $\mathrm{CE}$ for the $\mathrm{Sb}$-Te alloy, relaxing only the volume and the lattice parameters. The initial dataset contains the formation energies of all possible configurations of three, six, and nine layers in the hexagonal unit cell. Newly generated structures with a formation energy up or below the convex hull were systematically included in

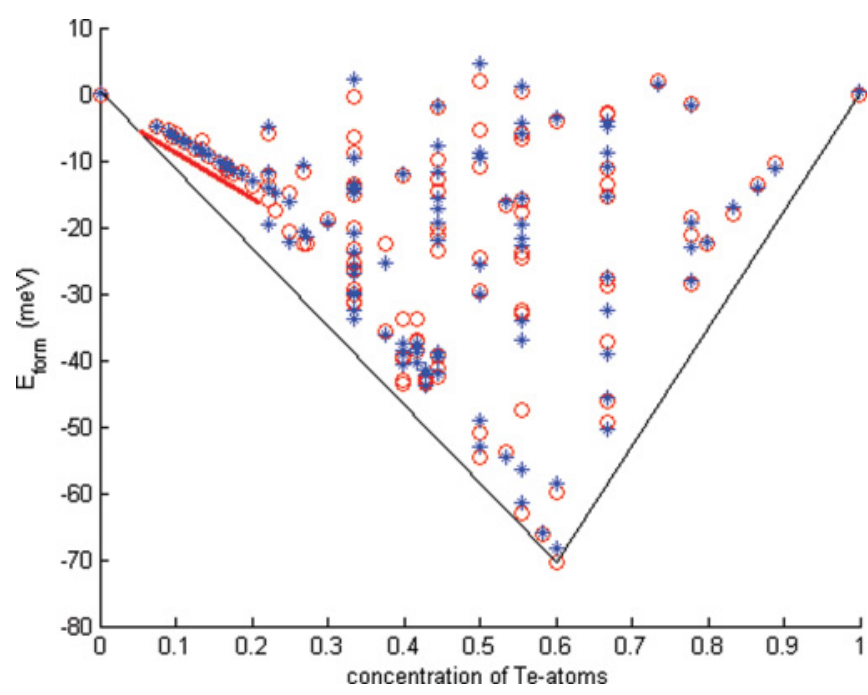

FIG. 2. (Color online) Formation energies before relaxing atomic positions calculated with VASP (CE) are denoted by red circles (blue stars). Convex hull is indicated by the solid black lines. Metastable compounds $(\mathrm{Sb})_{n}\left(\mathrm{Sb}_{2} \mathrm{Te}_{3}\right)_{m}$ are underlined by the red bar.

the $\mathrm{CE}$ and a converged result was obtained with 58 structures. Both the $a b$ initio and the predicted CE results are shown in Fig. 2. We notice that there is a very good agreement between both results; the LOOCV of this CE is $3.11 \mathrm{meV} /$ atom and includes 12 clusters with atom distances up to five interplanar distances and a maximum of three sites in a cluster. These clusters are the empty cluster, one-point cluster, all two-point clusters up to fifth neighbors, and all three-point clusters up to fourth neighbors. For details on this CE, see Supplemental Material. ${ }^{29}$ We clearly see that the most stable composition corresponds to $\mathrm{Sb}_{2} \mathrm{Te}_{3}$ and since no other structures are lying on the convex hull, all other compositions correspond to a two-phase region of $\mathrm{Sb}$ and $\mathrm{Sb}_{2} \mathrm{Te}_{3}$ for $x<0.6$, and $\mathrm{Te}$ and $\mathrm{Sb}_{2} \mathrm{Te}_{3}$ for $x>0.6$.

The converged CE shows that metastable compounds with large unit cells exist for high concentrations of $\mathrm{Sb}(x<0.2)$, underlined by the red bar in Fig. 2. Analyzing the structures in more detail reveals that the structure is built from successive layers of $\mathrm{Sb}$ next to successive units of $\mathrm{Sb}_{2} \mathrm{Te}_{3}$ and the composition of compounds with $0 \leqslant x \leqslant 0.6$ can therefore be written as $(\mathrm{Sb})_{n}\left(\mathrm{Sb}_{2} \mathrm{Te}_{3}\right)_{m}$, with $n, m=1,2,3, \ldots$ To further improve the $\mathrm{CE}$ we therefore included some of these structures in the dataset. We notice that for both $n$ even and odd the formation energy is close to the convex hull.

In the second step we also allowed the atomic positions to relax (in combination with a volume and lattice parameter optimization). To avoid that the atoms get trapped in local minima, all atoms were subjected to small random displacements from their initial fcc-lattice points. This relaxation results in alternating short and long Sb-Sb interlayer distances (a change of $3-10 \%$ ), thereby favoring the formation of $\mathrm{Sb}$ bilayers, which is reminiscent of the well-known bilayer formation in pure $\mathrm{Sb}$ and which results from a Peierls distortion. ${ }^{30}$ Moreover, the structures with an odd number of consecutive Sb layers become much less stable than those with an even number of consecutive Sb layers. 
In structures which contain the sequence $\mathrm{Te}-\mathrm{Sb}-\mathrm{Te}-\mathrm{Sb}$ $\mathrm{Te}$ (i.e., $\mathrm{Sb}_{2} \mathrm{Te}_{3}$ ) we observe a reduction in the interatomic distances (of 3-5\%) within this unit, whereas the distance between this $\mathrm{Sb}_{2} \mathrm{Te}_{3}$ sequence and the neighboring layers becomes larger.

Because of strong structural relaxations the standard CE method does not lead to a converged result, even with a large dataset (120 ab initio calculated structures). In particular, the generated CEs were never able to discriminate between structures with an even or odd number of successive Sb layers. Instead of further increasing the number of structures in our dataset [cf. with the case of $\mathrm{SnO}_{2-x}$ (Ref. 31)] or using more elaborate methods to account for the structural relaxations, ${ }^{32,33}$ we show that fast convergence can be achieved by considering a ternary CE. Here we account for the energetic differences between structures with an odd or even sequence of Sb layers (due to Peierls relaxation) by switching the occupation variable for $\mathrm{Sb}$ from 0 to -1 if the $\mathrm{Sb}$ layer is a part of a sequence of an even number $\mathrm{Sb}$ layers, while the occupation variable for Te remains 1.

In Fig. 3 the final $a b$ initio dataset is shown together with the $\mathrm{CE}$ predicted values. This $\mathrm{CE}$ has $\mathrm{LOOCV}=$ $10.88 \mathrm{meV} /$ atom, and includes seven clusters with atom distances up to five interplanar distances and a maximum of three sites in a cluster. These clusters are the empty cluster, one-point cluster, nearest-, third-, fourth-, and fifthneighbor clusters and a three-point cluster with fourth and fifth neighbors. A total of 72 structures up to 30 layers were considered. For details on this CE, see Supplemental Material. ${ }^{29}$ In Fig. 4 we used the CE to generate the formation energy of $10^{4}$ structures. $\mathrm{Sb}_{2} \mathrm{Te}_{3}$ clearly remains the most stable compound, but for the high Sb region with $0 \leqslant x \leqslant 0.6$ we observe a series of metastable multilayered structures with an even number of successive $\mathrm{Sb}$ layers adjacent to successive units of $\mathrm{Sb}_{2} \mathrm{Te}_{3}$. The general composition can therefore be written as $\left(\mathrm{Sb}_{2}\right)_{n}\left(\mathrm{Sb}_{2} \mathrm{Te}_{3}\right)_{m}$, in line with the experimental

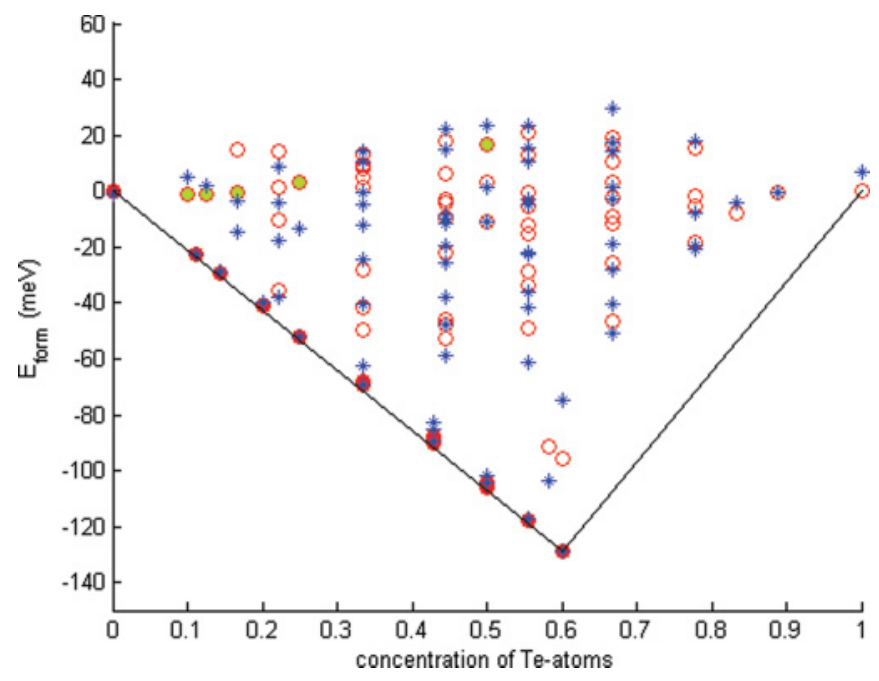

FIG. 3. (Color online) Formation energies after relaxation calculated with VASP (CE) are denoted by red circles (blue stars). Convex hull is indicated by the full black lines. Full red circles denote $\mathrm{Sb}_{n}\left(\mathrm{Sb}_{2} \mathrm{Te}_{3}\right)_{m}$ with $n$ even, and full green circles denote $\mathrm{Sb}_{n}\left(\mathrm{Sb}_{2} \mathrm{Te}_{3}\right)_{m}$ with $n$ odd.

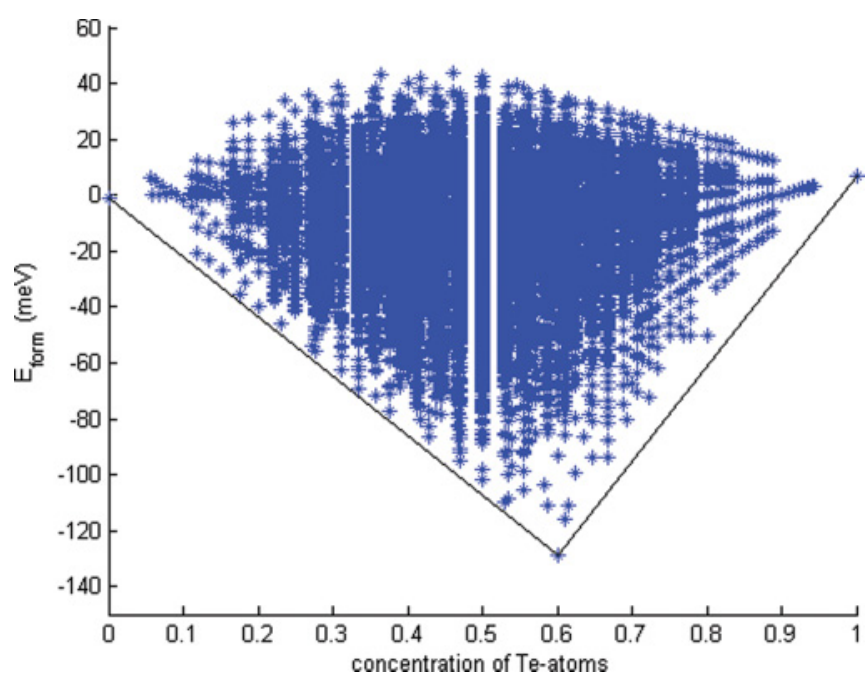

FIG. 4. (Color online) Predicted energies of all structures up to 18 layers.

data. ${ }^{9-11,13,34}$ Though our results show that $\left(\mathrm{Sb}_{2}\right)_{n}\left(\mathrm{Sb}_{2} \mathrm{Te}_{3}\right)_{m}$ compounds are metastable, it must be emphasized that we do not consider the effect of entropy (configurational, vibrational, or electronic), which might stabilize these structures at finite temperatures. These structures are only $1.13-4.18 \mathrm{meV}$ per atom removed from the convex hull.

The $\left(\mathrm{Sb}_{2}\right)_{n}\left(\mathrm{Sb}_{2} \mathrm{Te}_{3}\right)_{m}$ compounds have a lattice parameter $a$ between 4.33 and $4.36 \AA$ and $c$ depends on the number of layers in the unit cell.

For a given Te concentration $x,\left(\mathrm{Sb}_{2}\right)_{n}\left(\mathrm{Sb}_{2} \mathrm{Te}_{3}\right)_{m}$ becomes more stable for increasing $m$, with the most stable structure containing adjacent $\mathrm{Sb}_{2} \mathrm{Te}_{3}$ units. This is clearly demonstrated in Fig. 5, where lines corresponding to $m=1$ and $m=2$ (always with adjacent $\mathrm{Sb}_{2} \mathrm{Te}_{3}$ units) are added to that of the convex hull (the lowest line). Structures with larger $m$ are not only more stable, but also have a larger $c$ lattice parameter

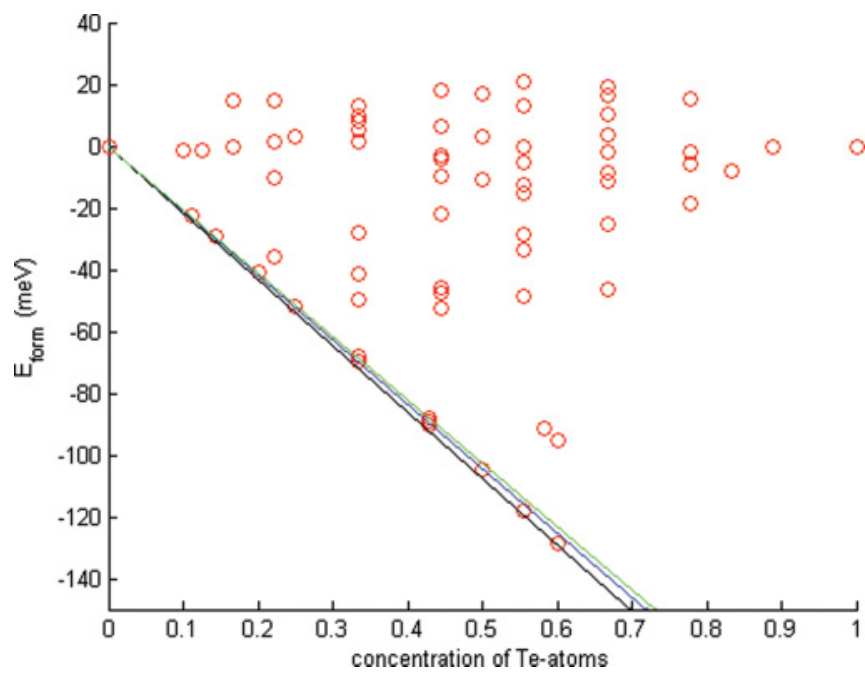

FIG. 5. (Color online) Ab initio calculated values are denoted by red circles. The green (upper) line connects the energies of structures with $m=1$, the blue (middle) line connects the energies of structures with $m=2$. The black line corresponds to the convex hull and connects the energies of the stable structures of pure $\mathrm{Sb}$ and $\mathrm{Sb}_{2} \mathrm{Te}_{3}$. 
(per layer), due to the large distances between two adjacent $\mathrm{Sb}_{2} \mathrm{Te}_{3}$ units. The fact that the most stable structures are obtained for successive $\mathrm{Sb}_{2} \mathrm{Te}_{3}$ units adjacent to successive $\mathrm{Sb}_{2}$ units shows that $\mathrm{Sb}-\mathrm{Te}$ alloys prefer to segregate into a pure $\mathrm{Sb}$ part and a pure $\mathrm{Sb}_{2} \mathrm{Te}_{3}$ part.

The possibility of competing stable nonlayered structures was investigated by using a three-dimensional CE as implemented in the ATAT code. ${ }^{35}$ However, no stable nonlayered $\mathrm{Sb}_{1-x} \mathrm{Te}_{x}$ alloys were found, using an underlying fcc lattice and unit cells up to 12 atoms. It should be noted that the layered structures $\left(\mathrm{Sb}_{2}\right)_{n}\left(\mathrm{Sb}_{2} \mathrm{Te}_{3}\right)_{m}$ did not show up in the three-dimensional $\mathrm{CE}$ because of the large unit cells involved thereby justifying our one-dimensional approach.

Although the Sb-Te phase diagram exhibits intermediate single phases $\delta$ and $\gamma$, and the $\delta+\gamma$ two-phase region, our $T=0 \mathrm{~K}$ results do not show any qualitative difference between the various concentration regions. This might be due to the fact that our calculations do not include entropy contributions. On the other hand, our findings are in line with recent experimental results, which demonstrated the existence of several $\mathrm{Sb}$-Te compounds with a very long stacking period [up to $c \sim 110$ $\AA$ (Ref. 11)] consisting of a stacking of $\mathrm{Sb}_{2} \mathrm{Te}_{3}$ units followed by $\mathrm{Sb}$ bilayers along the whole composition range from 0 to 60 at. $\%$ Te. The compound $\mathrm{Sb}_{3} \mathrm{Te}_{2}$ (40 at. \% Te), which belongs to the $\delta+\gamma$ region is found to consist of one single phase built up from $\mathrm{Sb}_{2} \mathrm{Te}_{3}$ units and $\mathrm{Sb}$ bilayers. ${ }^{12}$ Compounds with general composition $\mathrm{Sb}_{2 n} \mathrm{Te}_{3}(n=3,4, \ldots, 8),{ }^{11}$ which belong to the $\delta$ or $\gamma$ phase or to the two-phase region $\mathrm{Sb}+\delta$, and compounds $\mathrm{Sb}_{64} \mathrm{Te}_{36}, \mathrm{Sb}_{72} \mathrm{Te}_{28}$ and $\mathrm{Sb}_{76} \mathrm{Te}_{24}$ (Ref. 13) (all belonging to the $\delta$ phase) reveal no phase separation but consist of successive units of $\mathrm{Sb}_{2} \mathrm{Te}_{3}$ and $\mathrm{Sb}_{2}$.

In the $\left(\mathrm{Sb}_{2}\right)_{n}\left(\mathrm{Sb}_{2} \mathrm{Te}_{3}\right)_{m}$ structures the distance for within the Sb bilayers is between 1.54 and $1.57 \AA$ when they form bilayers, while the distance between such bilayers is much larger, between 2.22 and $2.29 \AA$. For structures $\mathrm{Sb}_{n}\left(\mathrm{Sb}_{2} \mathrm{Te}_{3}\right)_{m}$ with $n$ odd, the Sb layers also try to form bilayers. For $n$ large, we can see the formation of bilayers near the $\mathrm{Sb}_{2} \mathrm{Te}_{3}$ unit, while this is no longer observed far away from the $\mathrm{Sb}_{2} \mathrm{Te}_{3}$ unit. The difference between $n$ even or odd is illustrated in Fig. 6 for $\mathrm{Sb}_{16}\left(\mathrm{Sb}_{2} \mathrm{Te}_{3}\right)$ and $\mathrm{Sb}_{13}\left(\mathrm{Sb}_{2} \mathrm{Te}_{3}\right)$. Regarding the distance between the $(\mathrm{Sb})_{n}$ part and the $\left(\mathrm{Sb}_{2} \mathrm{Te}_{3}\right)_{m}$ block values between 2.36 and $2.45 \AA$ are found for $n$ even. For $n$ odd a much larger variation is observed: the distance between the $\mathrm{Sb}$ layer and the $\mathrm{Sb}_{2} \mathrm{Te}_{3}$ block in $\mathrm{Sb}\left(\mathrm{Sb}_{2} \mathrm{Te}_{3}\right)$ is $1.84 \AA$ whereas a distance of $2.39 \AA$ is found in $\mathrm{Sb}_{25}\left(\mathrm{Sb}_{2} \mathrm{Te}_{3}\right)$. The total distance from the first Te layer till the last Te layer in $\mathrm{Sb}_{2} \mathrm{Te}_{3}$ is in all cases between 7.3 and 7.5 $\AA$. The separation between adjacent $\mathrm{Sb}_{2} \mathrm{Te}_{3}$ units is between 2.65 and $2.69 \AA$, consistent with a van der Waals type of bonding.

To illustrate the effect of the vdW-DF functional, Table I shows for four structures (pure $\mathrm{Sb}, \mathrm{Sb}_{2} \mathrm{Te}_{3}, \mathrm{Sb}_{2} \mathrm{Te}$, and $\mathrm{SbTe}$ ) the experimental, PBE calculated, and optB86b calculated distances between layers where the bonding is of weak vdW type: between the $\mathrm{Sb}$ bilayers ( $\mathrm{Sb}-\mathrm{Sb}$ ), between the $\mathrm{Sb}_{2} \mathrm{Te}_{3}$ units (Te-Te), and between the bilayers and the $\mathrm{Sb}_{2} \mathrm{Te}_{3}$ units (Sb-Te). In addition we also list the $c$ parameter. From this table we observe that the optB86b functional systematically improves the interlayer distances (there is an error of $0-5 \%$ from the experimental value, compared with an error of $3-13 \%$ for the PBE calculated distances) thereby leading to better

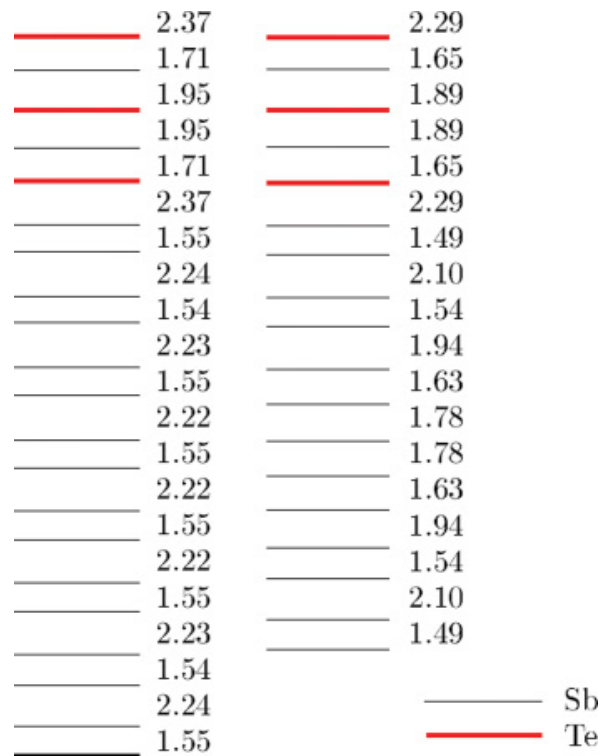

FIG. 6. (Color online) The interlayer distances (in $\AA$ ) for $\mathrm{Sb}_{16}\left(\mathrm{Sb}_{2} \mathrm{Te}_{3}\right)$ and $\mathrm{Sb}_{13}\left(\mathrm{Sb}_{2} \mathrm{Te}_{3}\right)$.

values for the $c$ parameter (the non-vdW distances depend much less on the choice of the functional). Though the vdW-DF improves the interlayer distances in a significant way, the relative stability of the structures with respect to the convex hull is hardly influenced by the use of the vdW-DF.

\section{B. Electronic properties \\ 1. Density of states}

In this section we discuss the band structure and density of states (DOS) of the homologous series of metastable structures $\left(\mathrm{Sb}_{2}\right)_{n}\left(\mathrm{Sb}_{2} \mathrm{Te}_{3}\right)_{m}$ found in the previous section.

The electronic structure of $\mathrm{Sb}_{2} \mathrm{Te}_{3}$ has been intensively investigated in the (recent) past both because of its thermoelectric properties ${ }^{3}$ and its behavior as a topological insulator. ${ }^{1,2}$ We found $\mathrm{Sb}_{2} \mathrm{Te}_{3}$ to be a semiconductor with a band gap of $0.16 \mathrm{eV}$. This result is in agreement with other DFT results. ${ }^{36}$ The spin-orbit coupling was not included in our calculations, but is known to increase the band gap to $0.28 \mathrm{eV}^{36}$ The top

TABLE I. Interlayer vdW distances and $c$ parameter (both in $\AA$ ) for pure $\mathrm{Sb}, \mathrm{Sb}_{2} \mathrm{Te}_{3}, \mathrm{Sb}_{2} \mathrm{Te}$, and $\mathrm{SbTe}$ obtained from experimental values (Ref. 6), values from PBE calculations, and vdW calculations.

\begin{tabular}{lcrrr}
\hline \hline & & Expt. & PBE & optB86b \\
\hline Pure $\mathrm{Sb}$ & $\mathrm{Sb}-\mathrm{Sb}$ & 2.24 & 2.30 & 2.21 \\
& $c$ & 11.22 & 11.48 & 11.26 \\
$\mathrm{Sb}_{2} \mathrm{Te}_{3}$ & $\mathrm{Te}-\mathrm{Te}$ & 2.83 & 3.07 & 2.68 \\
& $c$ & 30.46 & 31.40 & 30.25 \\
$\mathrm{Sb}_{2} \mathrm{Te}$ & $\mathrm{Sb}-\mathrm{Sb}$ & 2.34 & 2.45 & 2.27 \\
& $\mathrm{Sb}-\mathrm{Te}$ & 2.42 & 2.52 & 2.38 \\
& $c$ & 17.63 & 17.83 & 17.48 \\
$\mathrm{SbTe}$ & $\mathrm{Sb}-\mathrm{Te}$ & 2.44 & 2.58 & 2.43 \\
& $\mathrm{Te}-\mathrm{Te}$ & 2.68 & 3.03 & 2.65 \\
& $c$ & 23.90 & 24.49 & 23.82 \\
\hline \hline
\end{tabular}




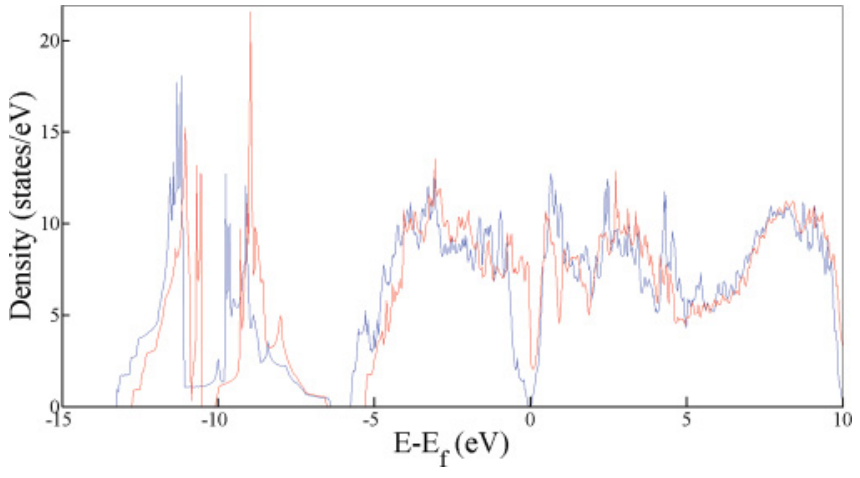

FIG. 7. (Color online) DOS of unrelaxed (red) and relaxed (blue) SbTe.

of the valence band mainly consists of Te states, whereas the character of the conduction band is mainly $\mathrm{Sb}$.

Pure $\mathrm{Sb}$ is a semimetal; each $\mathrm{Sb}$ atom in a bilayer has three nearest neighbors with which it forms strong covalent bonds. The bonding between the different bilayers is of weak van der Waals type.

Our original binary $\mathrm{CE}$ failed to converge because of the strong structural relaxations. In particular, the binary $\mathrm{CE}$ could not discriminate between structures $\mathrm{Sb}_{n}\left(\mathrm{Sb}_{2} \mathrm{Te}_{3}\right)_{m}$ with $n$ even or odd. When considering for example $\left(\mathrm{Sb}_{2}\right)\left(\mathrm{Sb}_{2} \mathrm{Te}_{3}\right)_{2}$ (abbreviated as $\mathrm{SbTe}$ ), we see that before relaxation of the atomic positions this structure is metallic, whereas it turns into a semiconductor after relaxation (see Fig. 7). This metalinsulator transition, together with the Sb-bilayer formation, shows that also the multilayered systems $\mathrm{Sb}_{n}\left(\mathrm{Sb}_{2} \mathrm{Te}_{3}\right)_{m}$ undergo a Peierls transition.

However, compounds with an odd number of adjacent $\mathrm{Sb}$ layers remain metallic after relaxing the atomic positions with a high DOS at the Fermi level $\left(E_{\mathrm{f}}\right)$, as shown in Fig. 8 for $\mathrm{Sb}_{7}\left(\mathrm{Sb}_{2} \mathrm{Te}_{3}\right)$. The metal-insulator transition is hampered by the unavoidable frustration that occurs on forming Sb bilayers.

Since the stable compounds $\mathrm{Sb}$ and $\mathrm{Sb}_{2} \mathrm{Te}_{3}$ are semimetallic and semiconducting respectively, one expects a transition between 0 and 60 at. \% Te. In Fig. 9 we give the band structure of three (relaxed) multilayered structures with an increasing amount of $\mathrm{Sb}$ : $\left(\mathrm{Sb}_{2}\right)\left(\mathrm{Sb}_{2} \mathrm{Te}_{3}\right)_{2},\left(\mathrm{Sb}_{2}\right)_{2}\left(\mathrm{Sb}_{2} \mathrm{Te}_{3}\right)$, and $\left(\mathrm{Sb}_{2}\right)_{5}\left(\mathrm{Sb}_{2} \mathrm{Te}_{3}\right)$, abbreviated as $\mathrm{SbTe}, \mathrm{Sb}_{2} \mathrm{Te}$ and $\mathrm{Sb}_{4} \mathrm{Te}$, respectively. The compound $\mathrm{SbTe}$ is a semiconductor with a band gap of $0.029 \mathrm{eV}$, but both $\mathrm{Sb}_{2} \mathrm{Te}$ and $\mathrm{Sb}_{4} \mathrm{Te}$ are

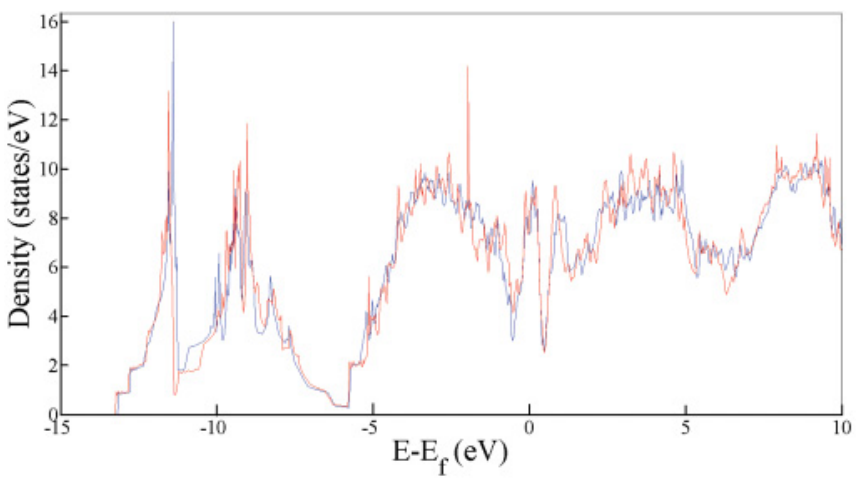

FIG. 8. (Color online) DOS of unrelaxed (red) and relaxed (blue) $\mathrm{Sb}_{7}\left(\mathrm{Sb}_{2} \mathrm{Te}_{3}\right)$.
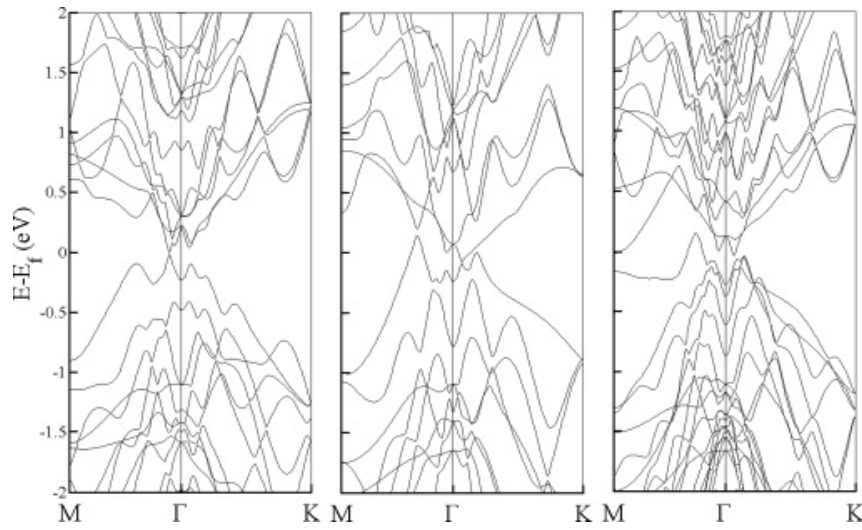

FIG. 9. Band structure of SbTe (left), $\mathrm{Sb}_{2} \mathrm{Te}$ (middle), $\mathrm{Sb}_{4} \mathrm{Te}$ (right).

semimetals. In all cases, the top of the valence band and the bottom of the conduction band are composed of $\mathrm{Sb}$ states coming from both the $\mathrm{Sb}$ bilayers and the $\mathrm{Sb}_{2} \mathrm{Te}_{3}$ units.

\section{Electron localization function}

In order to get a detailed picture of the chemical bonding in the layered Sb-Te structures, we have calculated the electron localization function (ELF) ${ }^{37,38}$ for the configuration $\mathrm{Sb}_{6}\left(\mathrm{Sb}_{2} \mathrm{Te}_{3}\right) \mathrm{Sb}_{5}\left(\mathrm{Sb}_{2} \mathrm{Te}_{3}\right)$, containing an odd and an even sequence of $\mathrm{Sb}$ layers, separated by an $\mathrm{Sb}_{2} \mathrm{Te}_{3}$ unit. The ELF ranges from 0 to 1 where the latter value corresponds to full localization and a value of 0.5 corresponds to the uniform electron gas. From Fig. 10 we see that the strongest covalent bonds occur between the Sb bilayers in the case of an even number of Sb layers. A weaker bond is found between the $\mathrm{Sb}$

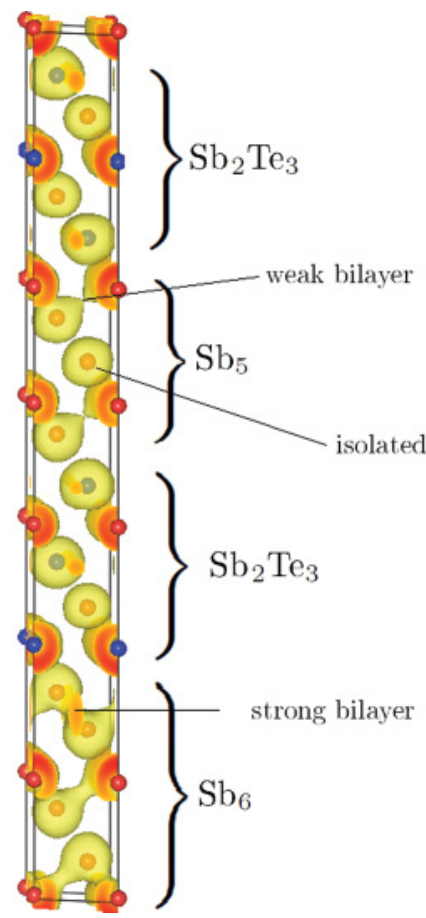

FIG. 10. (Color online) The ELF isosurfaces for relaxed $\mathrm{Sb}_{6}\left(\mathrm{Sb}_{2} \mathrm{Te}_{3}\right) \mathrm{Sb}_{5}\left(\mathrm{Sb}_{2} \mathrm{Te}_{3}\right)$ for a value 0.72 . Red: $\mathrm{Sb}$ atoms; blue: Te atoms. 
atoms of an odd number of layers. In the latter case the bilayer formation is frustrated which is also apparent from Fig. 6. In order of decreasing strength the other occurring covalent bonds are the bonding between $\mathrm{Te}$ and $\mathrm{Sb}$ occurring in the $\mathrm{Sb}_{2} \mathrm{Te}_{3}$ unit, the bonding between successive $\mathrm{Sb}$ bilayers, and finally the bonding between $\mathrm{Sb}$ layers and $\mathrm{Sb}_{2} \mathrm{Te}_{3}$ units.

\section{CONCLUSION}

The stability (at $T=0 \mathrm{~K}$ ) of multilayered $\mathrm{Sb}$-Te alloys has been studied by a combination of a ternary one-dimensional $\mathrm{CE}$ and accurate first-principles calculations. Due to the strong structural relaxations accompanying the Peierls transition a converging binary $\mathrm{CE}$ of the formation energy cannot be realized with a reasonable number of input structures $(\sim 100)$. In addition to the stable compound $\mathrm{Sb}_{2} \mathrm{Te}_{3}$ a homologous series of metastable layered compounds was found composed of units of $\mathrm{Sb}$ bilayers and $\mathrm{Sb}_{2} \mathrm{Te}_{3}$ units. The Peierls transition, with its $\mathrm{Sb}$-bilayer formation, occurs not just in the pure $\mathrm{Sb}$ and $\mathrm{Te}$, but is a generic feature that occurs across the whole composition range of V-VI compounds. The Peierls transition gives rise to particular structure types in which multilayer motifs play a dominant role.

For a given amount of $\mathrm{Sb}$ (or $\mathrm{Te}$ ) the most stable structure corresponds to the situation where (1) Sb bilayers are grouped together, (2) $\mathrm{Sb}_{2} \mathrm{Te}_{3}$ units are grouped together, (3) the largest number of adjacent $\mathrm{Sb}_{2} \mathrm{Te}_{3}$ units is found. Our $T=0 \mathrm{~K}$ results do not show the presence of the $\delta, \gamma, \delta+\gamma$ phase regions in the range $0<x<60$ at. $\%$ Te, which are reported in the phase diagram. ${ }^{7}$

Metastable structures $\left(\mathrm{Sb}_{2}\right)_{n}\left(\mathrm{Sb}_{2} \mathrm{Te}_{3}\right)_{m}(n, m=1,2,3, \ldots)$ with an $\mathrm{Sb}$ amount close to that of $\mathrm{Sb}_{2} \mathrm{Te}_{3}$ are semiconducting with a band gap decreasing (starting from the $\mathrm{Sb}_{2} \mathrm{Te}_{3}$ value of $0.16 \mathrm{eV}$ ) with increasing Sb concentration. For $x$ between 0.4 and 0.5 the layered structures become semimetallic. In particular $\mathrm{Sb}_{2} \mathrm{Te}$ is semimetallic, whereas $\mathrm{SbTe}$ is semiconducting. Adding one extra $\mathrm{Sb}$ layer (or an odd number of layers) to the $\left(\mathrm{Sb}_{2}\right)_{n}$ unit results in an unstable metallic structure with a high density of states at the Fermi energy.

In this work we have shown that first-principles electronic structure calculations yield insight into the microscopic origin of the stability of $\mathrm{Sb}$-Te alloys, thereby providing a first step toward a more detailed study of thermodynamic stability at $T \neq 0 \mathrm{~K}$ and toward the study of doped $\mathrm{Sb}$-Te systems.

\section{ACKNOWLEDGMENTS}

We gratefully acknowledge financial support from the IWT-Vlaanderen through the ISIMADE project, the FWOVlaanderen through Project No. G.0191.08, and the BOF-NOI of the University of Antwerp. K.G. thanks the University of Antwerp for a Ph.D. fellowship. This work was carried out using the HPC infrastructure at the University of Antwerp (CalcUA), a division of the Flemish Supercomputer Center VSC, supported financially by the Hercules Foundation. *kirsten.govaerts@ua.ac.be
${ }^{\dagger}$ m.h.f.sluiter@tudelft.nl
${ }^{\ddagger}$ bart.partoens@ua.ac.be
${ }^{\S}$ dirk.lamoen@ua.ac.be

${ }^{1}$ H. J. Zhang, C. X. Liu, X. L. Qi, X. Dai, Z. Fang, and S. C. Zhang, Nat. Phys. 5, 438 (2009).

${ }^{2}$ D. Hsieh, Y. Xia, D. Qian, L. Wray, F. Meier, J. H. Dil, J. Osterwalder, L. Patthey, A. V. Fedorov, H. Lin, et al., Phys. Rev. Lett. 103, 146401 (2009).

${ }^{3}$ R. Venkatasubramanian, E. Siivola, T. Colpitts, and B. O'Quinn, Nature (London) 413, 597 (2001).

${ }^{4}$ M. Wuttig and N. Yamada, Nat. Mater. 6, 824 (2007).

${ }^{5}$ M. H. Lankhorst, B. W. Ketelaars, and R. A. Wolters, Nat. Mater. 4, 347 (2005).

${ }^{6}$ P. Villars and L. D. Calvert, Pearson's Handbook of Crystallographic Data for Intermetallic Phases (ASM International, Materials Park, OH, 1991).

${ }^{7}$ G. Ghosh, J. Phase Equilib. 15, 349 (1994).

${ }^{8}$ N. K. Abrikosov and M. M. Stasova, Inorg. Mater. 21, 1758 (1985).

${ }^{9}$ L. E. Shelimova, O. G. Karpinskii, M. A. Kretova, V. I. Kosyakov, V. A. Shestakov, V. S. Zemskov, and F. A. Kuznetsov, Inorg. Mater. 36, 768 (2000).

${ }^{10}$ P. M. Imamov and S. A. Semiletov, Kristallografiya 15, 972 (1970).

${ }^{11} \mathrm{~K}$. Kifune, Y. Kubota, T. Matsunaga, and N. Yamada, Acta Crystallogr. Sect. B 61, 492 (2005).

${ }^{12}$ C. W. Sun, J. Y. Lee, M. S. Youm, and Y. T. Kim, Phys. Stat. Sol. (RRL) 1, R25 (2007).
${ }^{13}$ C. W. Sun, J. Y. Lee, M. S. Youm, and Y. T. Kim, Jpn. J. Appl. Phys. 45, 9157 (2006).

${ }^{14}$ N. Frangis, S. Kuypers, C. Manolikas, G. Van Tendeloo, J. Van Landuyt, and S. Amelinckx, J. Solid State Chem. 84, 314 (1990).

${ }^{15}$ V. Agafonov, N. Rodier, R. Cedin, R. Bellissent, C. Bergman, and J. P. Gaspard, Acta Crystallogr. Sect. C 47, 1141 (2002).

${ }^{16}$ D. de Fontaine, Solid State Phys. 47, 33 (1994).

${ }^{17}$ J. M. Sanchez, F. Ducastelle, and D. Gratias, Physica A 128, 334 (1984).

${ }^{18}$ J. W. D. Connolly and A. R. Williams, Phys. Rev. B 27, 5169 (1983), ${ }^{19}$ M. H. F. Sluiter and Y. Kawazoe, Phys. Rev. B 71, 212201 (2005).

${ }^{20}$ A. van de Walle and G. Ceder, J. Phase Equilib. 23, 348 (2002).

${ }^{21}$ M. H. F. Sluiter, Y. Watanabe, D. de Fontaine, and Y. Kawazoe, Phys. Rev. B 53, 6137 (1996).

${ }^{22}$ M. H. F. Sluiter and Y. Kawazoe, Phys. Rev. B 68, 085410 (2003).

${ }^{23}$ C. Wolverton and D. de Fontaine, Phys. Rev. B 49, 8627 (1994).

${ }^{24}$ G. Kresse and J. Furthmüller, Comput. Mater. Sci. 6, 15 (1996).

${ }^{25}$ G. Kresse and J. Furthmüller, Phys. Rev. B 54, 11169 (1996).

${ }^{26}$ J. P. Perdew, K. Burke, and M. Ernzerhof, Phys. Rev. Lett. 77, 3865 (1996).

${ }^{27}$ J. Klimes, D. R. Bowler, and A. Michaelides, Phys. Rev. B 83, 195131 (2011).

${ }^{28}$ H. J. Monkhorst and J. D. Pack, Phys. Rev. B 13, 5188 (1976).

${ }^{29}$ See Supplemental Material at http://link.aps.org/supplemental/ 10.1103/PhysRevB.85.144114 for a table containing the structures used for the $\mathrm{CE}$ with their ab initio calculated energies, and the clusters of this CE with their ECIs. 
${ }^{30}$ K. J. Chang and M. L. Cohen, Phys. Rev. B 33, 7371 (1986).

${ }^{31}$ A. Seko, A. Togo, F. Oba, and I. Tanaka, Phys. Rev. Lett. 100, 045702 (2008).

${ }^{32}$ D. B. Laks, L. G. Ferreira, S. Froyen, and A. Zunger, Phys. Rev. B 46, 12587 (1992).

${ }^{33}$ O. Shchyglo, A. Diaz-Ortiz, A. Udyansky, V. N. Bugaev, H. Reichert, H. Dosch, and R. Drautz, J. Phys.: Condens. Matter. 20, 045207 (2008).
${ }^{34}$ M. S. Youm, Y. T. Kim, Y. H. Kim, and M. Y. Sung, Phys. Status Solidi (a) 205, 1636 (2008).

${ }^{35}$ A. van de Walle, M. Asta, and G. Ceder, CALPHAD: Comput. Coupling Phase Diagrams Thermochem. 26, 539 (2002).

${ }^{36}$ T. Thonhauser, T. J. Scheidemantel, J. O. Sofo, J. V. Badding, and G. D. Mahan, Phys. Rev. B 68, 085201 (2003).

${ }^{37}$ B. Silvi and A. Savin, Nature (London) 371, 683 (1994).

${ }^{38}$ A. D. Becke and K. E. Edgecombe, J. Chem. Phys. 92, 1990 (1990). 